Espacio y Desarrollo $\mathrm{N}^{\circ} 33,2019$, pp. 47-65 (ISSN 1016-9148)

https://doi.org/10.18800/espacioydesarrollo.201901.003

\title{
Geografías afectivas del pueblo kukama, Amazonía peruana
}

\author{
Daniel Fernandes Moreira \\ Grupo de Antropología Amazónica, Pontificia Universidad Católica del Perú \\ a20143860@pucp.pe \\ Marco Ramírez Colombier \\ Grupo de Antropología Amazónica, Pontificia Universidad Católica del Perú \\ mramirezc@pucp.pe
}

Fecha de recepción: 15 de abril 2019

Fecha de aceptación: 24 de mayo 2019

\section{Resumen}

La antropología amazónica contemporánea, en diálogo interdisciplinario con la arqueología, los estudios ecológicos, el derecho, la genética y la geografía, entre otras ciencias, abre paso a caracterizaciones del entorno amazónico que se alejan del discurso geográfico naturalista que guio las empresas de colonización de esta región. Estas nuevas perspectivas cobran especial relevancia en el contexto de crisis ecológica que caracteriza el Antropoceno, donde los pueblos indígenas buscan defender sus territorios frente a los proyectos extractivistas y de infraestructura. A través de la identificación de los modos de interacción que los kukama, un pueblo indígena del tronco tupí-guaraní, sostienen con espacios fluviales y terrestres propios del ecosistema de la llanura amazónica, encontramos procesos de domesticación y transformación de estos en territorios vitales. Consideramos que estas interacciones, basadas en las experiencias corporales, sensoriales y emocionales, configurarían una geografía afectiva que nos alienta a profundizar en los debates actuales de la geografía ambiental.

Palabras clave: Amazonía, geografía afectiva, pueblos indígenas, territorio, llanura amazónica.

\section{Affective geographies of the Kukama people, Peruvian Amazon}

\section{Abstract}

Contemporary Amazonian anthropology, in an interdisciplinary dialogue with archeology, ecological studies, law, genetics and geography, among other sciences, opens the way for characterizations of the Amazonian environment that move away from the naturalistic geographical discourse that guided the colonization of this region. These new perspectives are of particular interest in the context of the ecological crisis that characterizes the Anthropocene, 
where indigenous peoples seek to defend their territories against extractive and infrastructure projects. Through the analysis of the modes of interaction in which the Kukama, a tupiguarani indigenous people, engage with fluvial and ground spaces typical of the ecosystem of the Amazonian floodplain, we find processes of domestication and transformation of these spaces into vital territories. We consider that these interactions, based on bodily, sensory and emotional experiences, could configure an affective geography that encourages us to delve into current debates within environmental geography.

Keywords: Affective geography, Amazonia, Amazonian floodplain, indigenous peoples, territory.

\section{INTRODUCCIÓN}

La presente investigación tiene el propósito de verificar, a partir del punto de vista del pueblo indígena kukama, sentidos adscritos a determinadas prácticas espaciales que configurarían una geografía afectiva de la llanura de inundación amazónica. De esta manera, aportaremos al análisis de aquello que entendemos como la geografía ambiental de este ecosistema ubicado a orillas del río, y proporcionaremos pistas para comprender las dinámicas de ordenamiento de agentes y entornos en la Amazonía en un contexto de disputas territoriales.

Un discurso ampliamente difundido sobre la geografía del Perú ha representado a la Amazonía como una región carente de presencia humana significativa, cuyos habitantes nativos vivirían en condición de salvajes. Al mismo tiempo, la Amazonía ha sido imaginada como un entorno rico en recursos naturales, disponibles para su explotación al servicio del desarrollo nacional. Ambas narrativas convergen en una caracterización estereotipada de la Amazonía como un entorno natural homogéneo (Vadjunec, Schmink y Greiner, 2011), donde las sociedades indígenas estarían desprovistas de cultura e historicidad. Este discurso, fortalecido al servir de sustento a proyectos de colonización de la Amazonía en los siglos XIX y XX, continúa vigente en la discusión política del siglo XXI sobre el modelo de desarrollo socioeconómico basado en proyectos de extracción forestal, minera y petrolera, y en iniciativas de infraestructura para el transporte terrestre y fluvial (Espinosa, 2009; Ames, 2010).

Al margen de este discurso dominante, los pueblos indígenas amazónicos han desarrollado sus propios procesos de fortalecimiento de sus autonomías gracias a su capacidad para ejercer un control autónomo sobre los espacios que habitan y los recursos presentes en estos. De esta manera, se ha formulado el concepto de territorio indígena, que ha pasado de describir extensiones de tierras demarcadas a englobar un tejido de relaciones materiales y simbólicas entre agentes humanos, no humanos y los elementos de sus entornos (Surrallés, 2009). Este concepto ocupa un lugar central en los diálogos con los Estados, sobre todo a partir de la adopción de la Declaración de 
Naciones Unidas sobre los Derechos de los Pueblos Indígenas, que reconoce el derecho de estos pueblos a la libre determinación (artículos 3 y 4) y, con ello, legitima en el derecho internacional las iniciativas de los pueblos indígenas para desarrollar conceptos y prácticas propios sobre el espacio y los recursos (Espinosa, 2010), que, muchas veces, desbordan los modelos de territorialidad establecidos por los Estados (Surrallés, 2009). El territorio indígena ha sido visibilizado, entonces, como un concepto cosmopolítico (De la Cadena, 2010) que desafía los límites establecidos por la ciencia y la política de la modernidad, y a través del cual las cosmologías indígenas y sus conocimientos cosmogeográficos se introducen en la discusión pública.

Influenciada por desarrollos teóricos como el perspectivismo amerindio (Viveiros de Castro, 1998) o la ecología simbólica (Descola, 1996), la antropología amazónica contemporánea ha dedicado grandes esfuerzos a reflexionar sobre las teorías espacioterritoriales indígenas, y ha cuestionado la distinción occidental entre naturaleza y cultura, y, de manera paralela, entre los entornos naturales y los culturales. Como producto de esas reflexiones, encontramos nociones ontológicas como la de «territorio como cuerpo» (Echéverri, 2004); conceptos jurídico-políticos como el de «territorios integrales» (Garra y Riol, 2014); iniciativas de ordenamiento del entorno o «manejo del mundo» (Cayón, 2014); o métodos de relacionamiento ecológico a partir de la textualidad (Santos-Granero, 1998; Uzendoski, 2012), el movimiento (Zanotti, 2018) y la percepción (Surrallés, 2004).

Esta corriente antropológica dialoga, a menudo, con disciplinas como el derecho (García Hierro, 2004) y la historia ecológica (Balée, 2013), mientras que su atención al dinamismo del entorno amazónico coincide con las agendas de investigación de la arqueología amazónica (Heckenberger y Neves, 2009), la arqueobotánica y la genética (Clement, Heckenberg, Braga Junqueira, Neves, Texeira y Woods, 2015), o la geografía cultural (Vadjunec, Schmink y Greiner, 2011). Estos desarrollos teóricos aportan a una nueva caracterización de la Amazonía como un entorno complejo, marcado por el constante movimiento de una diversidad de agentes que moldean la vida en la interacción de sus particulares devenires históricos, identidades, reflexividades, ritualidades y corporalidades.

La llanura de inundación es un ecosistema amazónico cuyo estudio contribuye a visibilizar este tipo de procesos. Se trata de las tierras aledañas a los principales ríos amazónicos, aptas para la práctica de los cultivos debido a los minerales que las aguas de estos ríos traen desde los Andes, pero que se inundan totalmente en la estación del invierno amazónico, lo que exige la adaptación de los estilos de vida de los pueblos que las ocupan (Meggers, 1976; Smith, 2011). Luego de la discusión entre Betty Meggers (1976), Donald Lathrap (1970) y Anna Roosevelt (1993) sobre el nivel de complejidad que las sociedades asentadas al margen de los ríos pudieron haber alcanzado, diversas investigaciones se han dedicado a registrar los medios de vida de estas 
comunidades, basados en los cultivos estacionales (Chibnik, 1994), la pesca (Rivas, 2004) y la movilidad como técnica de manejo de recursos (Padoch, Brondizio, Costa, Pinedo-Vasquez, Sears y Siqueira, 2008).

Las llanuras inundables de los ríos Marañón, Huallaga y Ucayali, en la Amazonía peruana, son el lugar de asentamiento de las comunidades del pueblo indígena kukama, perteneciente a la familia lingüística tupí-guaraní. El pueblo kukama está estrechamente identificado con la llanura de inundación, a la cual han adaptado su arquitectura y sus prácticas de cultivo, al mismo tiempo que han desarrollado una gran destreza para la pesca y la navegación, que es reconocida por los demás pueblos amazónicos (Rivas, 2004). De forma simétrica a su involucramiento permanente con el entorno fluvial, su cosmología gira en torno a las relaciones sentidas entre el universo subacuático, el terrestre y el celestial. Los kukama recibieron poca atención de la antropología durante el siglo XX, cuando se les caracterizó como un pueblo en proceso de aculturación (Stocks, 1981, los llamó «nativos invisibles»). A fines del siglo XX e inicios del XXI, surgió un movimiento etnopolítico kukama, cuyas organizaciones demandaron el reconocimiento de su derecho a aprovechar los recursos de la Reserva Nacional Pacaya Samiria, a la cual consideran su territorio ancestral. Las luchas del pueblo kukama por la reivindicación de su historia e identidad como pueblo, en un contexto de ocupación de sus territorios por proyectos extractivos, turísticos y de infraestructura, han concitado la atención de académicos y activistas, lo que se ha traducido en un creciente número de investigaciones en torno a su ontología y chamanismo (Rivas, 2011; Berjón y Cadenas, 2014), la interacción en/con el espacio fluvial (Campanera Reig, 2017; Ramírez Colombier, 2018; Fernandes Moreira y Ramírez Colombier, 2019), los procesos identitarios locales (Castro Ríos, 2015) y la contienda política con el Estado (Okamoto y Leifsen, 2012).

La presente investigación se enmarca también en los desarrollos conceptuales surgidos de intelectuales y activistas indígenas del pueblo kukama en torno a su posicionamiento en una cosmología plural (Tello Imaina, 2014, 2016; Acodecospat, Aidecos, Akubana, Huaynakana Damatawara Kana y Orpio, 2015). Estas reflexiones están movilizadas por la defensa de sus territorios frente a proyectos de gran escala, como la extracción petrolera en el Lote 8X y el proyecto de infraestructura Hidrovía Amazónica, que contempla alteraciones permanentes a los ecosistemas de los ríos Amazonas, Huallaga, Marañón y Ucayali para acelerar el transporte fluvial. Este pensamiento no solamente se dirige a conceptualizar lugares, espacios y territorios, sino que surge de la relación con ellos. Como señala Leonardo Tello Imaina: «Siempre se ha vivido pensando pero el proceso de Consulta Previa [sobre la Hidrovía Amazónicaa]

\footnotetext{
1 La Hidrovía Amazónica es un megaproyecto de infraestructura que pretende dragar los cauces de los ríos Amazonas, Huallaga, Marañón y Ucayali para facilitar la navegación de embarcaciones mayores. Los kukama prevén graves impactos sobre el ecosistema fluvial como consecuencia de este proyecto.
} 
ha llevado a algunas gentes a otros espacios que no son los suyos y a pensar solos, lejos de sus mujeres y maridos, lejos de sus hijos, lejos de sus comunidades» (2016, p. 8).

Asimismo, pretendemos dar continuidad a nuestras reflexiones en desarrollo, desde la antropología y la arqueología, en torno a las transformaciones generadas por la interacción entre personas y espacios terrestres y acuáticos (Ramírez Colombier, 2018); la relación de los kukama con la tierra, el entorno y la etnoarqueología (Fernandes Moreira, en preparación); y los procesos territoriales adaptados a la movilidad de los ríos (Fernandes Moreira y Ramírez Colombier, 2019). De esta manera, buscamos establecer una vinculación entre estos estudios y las nuevas teorías críticas surgidas en la geografía, como el concepto de geografía afectiva (Pile, 2009; Bristow, 2015), para construir una mirada interdisciplinaria a un espacio vital amenazado por procesos característicos del Antropoceno.

\section{Metodología}

La información presentada se recogió en la comunidad nativa kukama Dos de Mayo, ubicada a orillas de la cocha San Pablo, en la margen derecha del curso bajo del río Marañón, a ocho horas río arriba desde el centro urbano de Nauta. Su población es de alrededor de 150 habitantes.

El territorio de esta comunidad se ubica dentro de la Reserva Nacional Pacaya Samiria, por lo que no cuenta con titulación territorial. Sin embargo, la comunidad ha logrado derechos de aprovechamiento sobre los recursos de la reserva y sostiene acuerdos consuetudinarios con otras comunidades para el uso de recursos pesqueros. La comunidad es un centro de la actividad política organizativa kukama, ya que alberga la sede de la Asociación Cocama de Desarrollo y Conservación San Pablo de Tipishca (Acodecospat), federación que agrupa a comunidades kukama de la cuenca del Maranón.

El trabajo de campo etnográfico se realizó en ocho visitas a la comunidad, entre octubre de 2017 y marzo de 2019, tanto en el período de invierno como en el verano amazónico. Durante estas visitas, se observaron los patrones de asentamiento y distribución de los sembríos en el territorio de la comunidad; se entrevistó a las personas mayores acerca de sus historias personales de desplazamiento y las de sus padres y abuelos; y se realizaron recorridos por lugares significativos del territorio de la comunidad junto a los pescadores y autoridades comunales kukama, lo que permitió identificar y mapear puntos de referencia, donde existen apariciones de seres cosmológicos, vestigios de asentamientos antiguos y lugares privilegiados de pesca. Esta información de primera mano ha sido complementada por información secundaria y documental, como mapas producidos por los apus o jefes comunales. 
En algunos pueblos amazónicos, se han encontrado simetrías entre las concepciones del espacio en diferentes niveles, desde lo local micro hasta la totalidad macro del territorio (por ejemplo, entre los tikuna, véase Santos Angarita, 2013). Tomando esto en cuenta, consideramos que el análisis del movimiento y ubicación de los kukama sobre un espacio inmediato y domesticado como el de la comunidad opera bajo preceptos similares a aquellos que ordenan su concepción cosmológica.

\section{Reflexividades KUKAMAS SOBRe LA TIERRA, EL Río Y EL BOSQUE}

Las claves interpretativas académicas desarrolladas en las últimas décadas nos permiten afirmar que la Amazonía descrita en la etnohistoria (Carvajal, 2010 [1540]) está siendo reinterpretada a partir de nuevos aportes científicos.

Los datos académicos recolectados por investigaciones etnográficas demuestran sorprendentes redes y conexiones entre el escenario panamazónico de la llanura amazónica y las tierras bajas tropicales. Esas conexiones tejen redes de sociabilidades, complejidades y diversidades en las dinámicas transformativas que ocurren en los contextos sociales, ambientales y políticos, lo que nos llevó a plantear la reflexión acerca de la formación de una geografía afectiva. En esta concepción surgida del pensamiento indígena, las presencias de diversas categorías de "gentes» habitan una pluralidad de «mundos» ${ }^{2}$. Estas presencias son responsables de la configuración de diferentes modos de habitar el espacio (Fernandes Moreira, en preparación) entre los períodos de verano e invierno amazónicos.

Podemos decir que los kukama sostienen una intensa relación con el mundo, donde los sueños forman parte de sus vidas y moldean sus espacios, territorio y geografía. Esta relación permite, por ejemplo, la comunicación con los seres habitantes del río, entre los cuales se encuentran sus familiares sumergidos, lo cual genera una vinculación afectiva con el espacio acuático que atraviesa todos los aspectos de la vida. En la reflexividad kukama, o en una de sus concepciones de la creación del mundo (Rivas, 2011), los grandes ríos de la Amazonía aparecieron cuando el dios Kukama lanzó con sus brazos fuertes las flechas grandes y pequeñas (Huaymacari Tamani, 2016). Las flechas grandes que cayeron originaron los grandes ríos, como el Marañón, el Ucayali o el Huallaga, a modo de transfiguraciones de la gran serpiente. Y cuando las flechas menores lanzadas por el dios Kukama cayeron, surgieron los ríos menores, las quebradas y los cańos.

\footnotetext{
2 Leonardo Tello Imaina (2016), intelectual y activista kukama, menciona categorías de ser «gente»: existe relación entre los «espíritus-gente», «animales-gente», «plantas-gente», «peces-gente» y «aves-gente» (p. 8).
} 
Figura 1. Comunidad nativa Dos de Mayo, área de cocha en período de verano amazónico

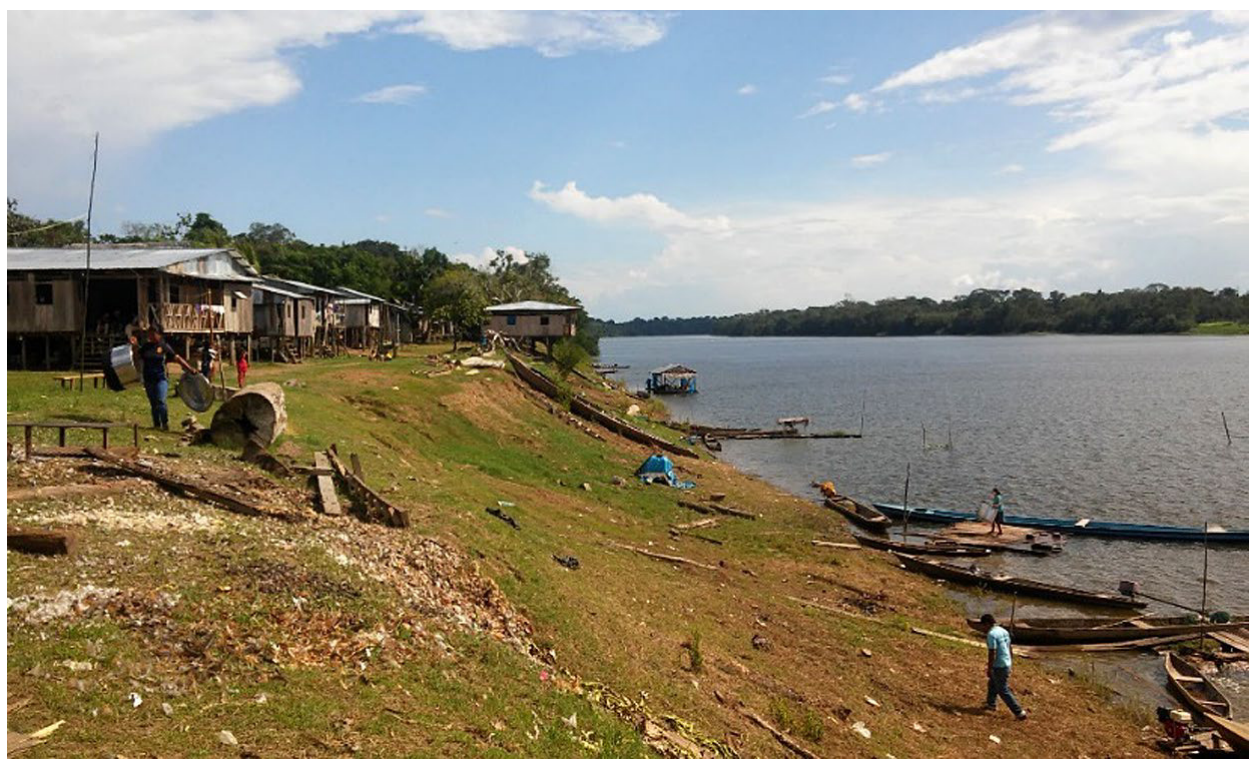

Fotografía: Marco Ramírez Colombier, octubre de 2017.

Figura 2. Comunidad nativa Dos de Mayo en período de invierno amazónico

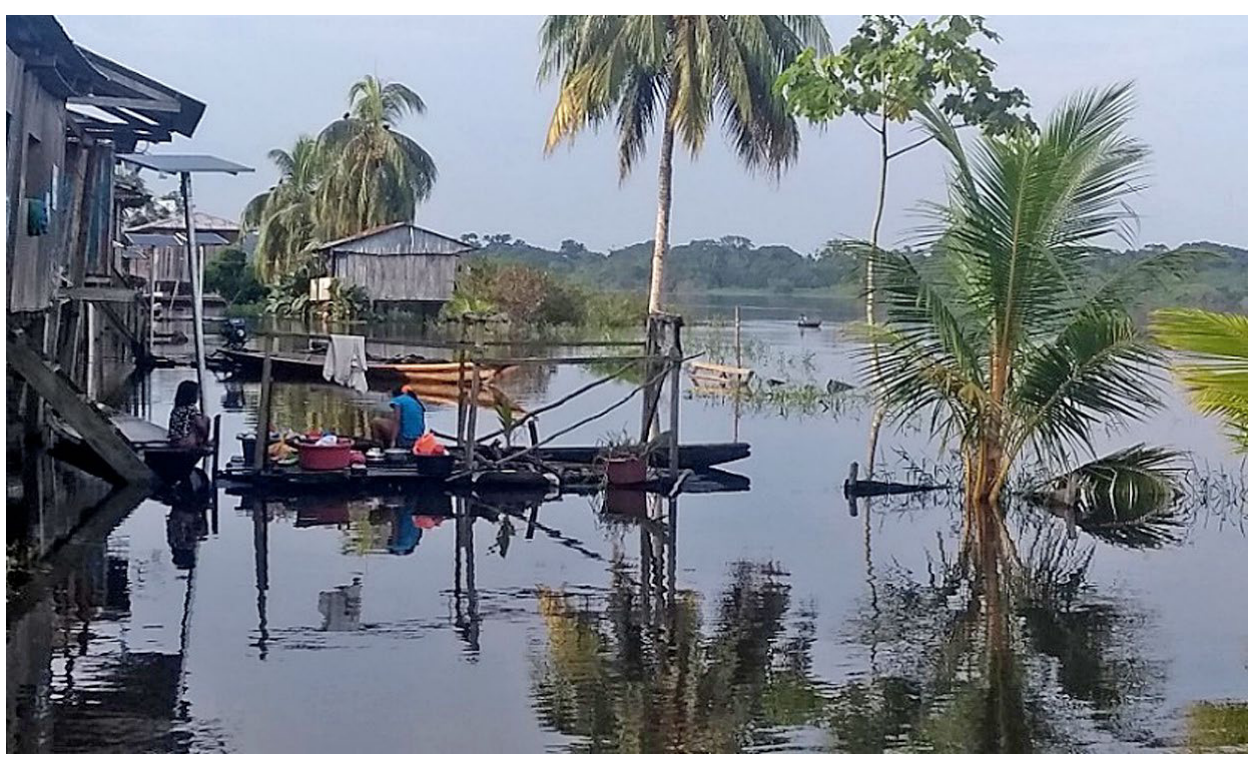

Fotografía: Daniel Fernandes Moreira, febrero de 2019. 
Figura 3. Vista del río Marañón en los bordes de la Reserva Nacional Pacaya Samiria. Salida de la comunidad Puerto Orlando en dirección a la comunidad Lisboa

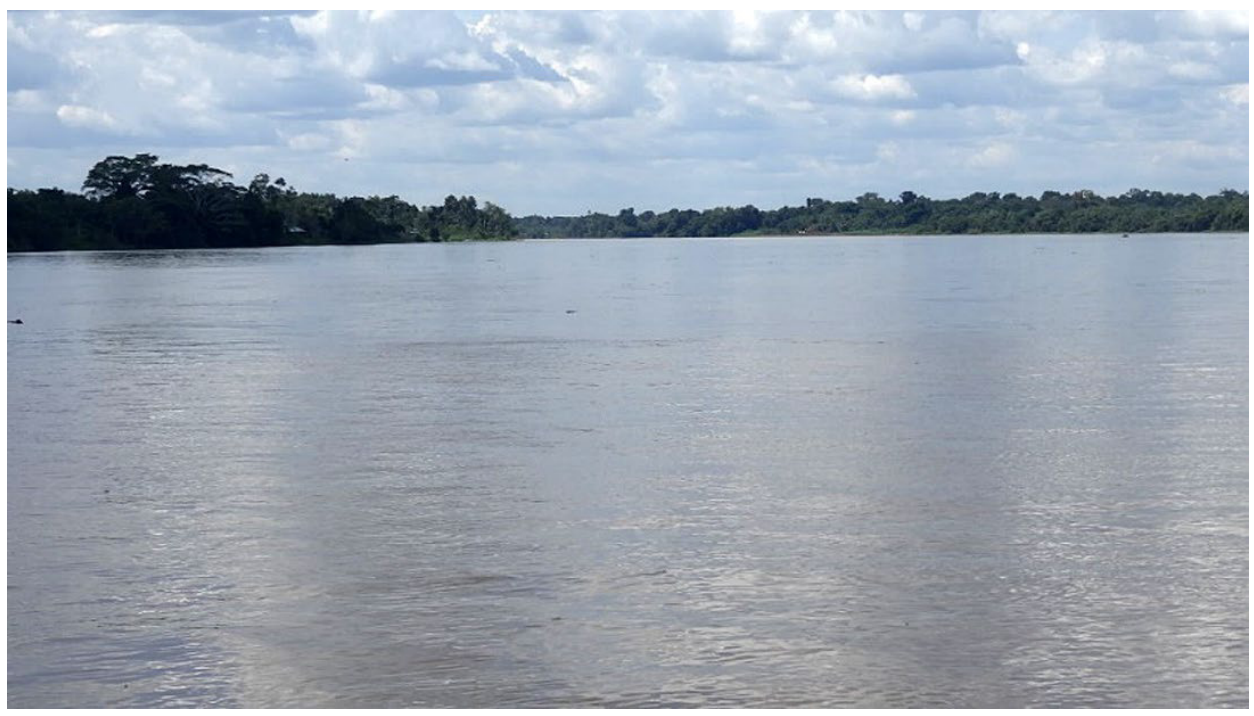

Fotografía: Daniel Fernandes Moreira, diciembre de 2018.

De esta manera, con el fin de comprender los espacios y los significados del concepto de "paisaje», tratamos de investigar el punto de vista de los kukama a partir de su cosmología indígena desarrollada en la Amazonía. Para esto, fue necesario apoyarnos en el marco teórico descrito líneas antes (Descola, 1986, 2014; Viveiros de Castro, 1996, 1998). Son de especial relevancia los escritos de Descola (2014), quien se pregunta si la noción de paisaje describe adecuadamente el proceso de antropización sufrido por los ecosistemas amazónicos, o si no sería preferible restringir el término "paisaje» a ocurrencias de transformación ambiental (Descola, 2014, p. 19). Esta pregunta es muy interesante para los estudios amazónicos y principalmente para la antropología, que promueve una nueva mirada a la relación de las personas y los entornos en el contexto de innumerables agresiones que ocurren contra las territorialidades indígenas.

En ese sentido, cuando pensamos en el paisaje, el río, los peces, la geografía, estamos articulados con los decires y la reflexividad kukama. Esta apunta a un discurso muy próximo a lo pensado por Descola, quien nos da un soporte teórico para el concepto de «transfiguración»: «[...] un cambio de apariencia deliberado al final del cual un sitio se vuelve un signo de otra cosa que lo que es, revelando así lo que potencialmente contenía» (Descola, 2014, p. 20). Eso nos hace reflexionar sobre los decires de los kukama, cuando afirman que el río «camina» y actúa con la purawa $a^{3}$, la responsable de

\footnotetext{
3 Dentro de la noción de territorialidad kukama, la purawa es descrita por los kukama como la serpiente madre, la boa que teje el territorio.
} 
configurar sus espacios territoriales y de dar origen al sedimento, las áreas encantadas, las casas, las tierras negras, las plantas y los bosques.

El bosque donde se desplazan los kukama está ubicado en un área ambiental no restringida (Neves, 2011) de la Amazonía. Este bosque amazónico, según las investigaciones contemporáneas, ha sido antropizado, domesticado (Balée y Erickson, 2006). Al considerar los estudios de la genética de las plantas (Clement et al., 2015) o las llamadas "maravillas amazónicas precolombinas» ${ }^{4}$ (Rostain y Jaimes Betancourt, 2017), tendremos evidencia de que esa domesticación está en actividad desde finales del Pleistoceno e inicios del Holoceno. Esto nos permite hacer articulaciones antropológicas refinadas y nos lleva a considerar estudios etnológicos como el de Rivas, quien menciona lo siguiente sobre la purawa como articuladora de la geografía kukama:

La modificación del curso del río durante la creciente, es percibido por los Kukama como el desplazamiento de la serpiente acuática. Asimismo, el curso del río representa su cuerpo y obedece a los avatares de su andar en la tierra, hecho análogo al continuo traslado del chamán sobre la tierra que nunca puede tener residencia fija en una comunidad. (Rivas, 2011, p. 93)

Tal vez por esto presenciamos un gran número de sitios arqueológicos en el área investigada (Morales Chocano, 2002), y por eso no descartamos, en esta descripción, los estudios de la prehistoria a partir de la arqueología (Karadimas, Lécrivain y Rostain, 2018) y de la etnoarqueología (Dillehay, 2007; Politis, 2007; Silva, 2014).

La industria artefactual ceramista de los utensilios presenta una fuerte relación con el manejo de alimentos y semillas, y con las poblaciones de plantas domesticadas y no domesticadas (Clement et al., 2015). Además, estos utensilios funcionan como reservorios de agua y alimentos, e incluso como urnas funerarias (Miller, 1987). Según un relato contado por el señor Víctor Canayo Pacaya, sabio kukama de la ciudad de Nauta y hablante de la lengua kukama, «las mujeres kukama iban hasta las orillas del río para buscar la greda o arcilla de colores blanca, amarilla, roja y gris. La corteza del árbol apacharama era usada para afirmar la alfarería y la tawa (greda) era utilizada como pintura negra».

Ese nuevo abordaje de interpretar el mundo se interconecta con los conceptos de la antropización; nos hace retornar a los presupuestos de Descola (2014), donde el proceso de antropización trae a la mente un cambio de perspectiva, «desde una naturaleza determinista hacia una naturaleza profundamente humanizada» (p. 19).

\footnotetext{
4 Concheros, montículos, camellones, tierras negras, geoglifos, megalitos y arte rupestre, según el volumen editado por Rostain y Jaimes Betancourt (2017).

5 Traducción hecha por la misma autora. El texto original en francés dice lo siguiente: «La modification du lit du fleuve au gré de la crue est perçue symboliquement pas les Cocama comme le déplacement du serpent d'eau. Le cours du fleuve représente son corps et suit les hauts et les bas de sa trace sur la terre, analogue au transfert continu du chamane sur la terre qui ne peut jamais posséder de résidence permanente dans une communauté» (Rivas, 2011, p. 93).
} 
Según Descola, este tipo de aporte en la teoría antropológica constituye un considerable progreso en la inteligibilidad de las interacciones complejas entre humanos y no humanos (2014, p. 19), que interactúan, en el caso que describimos, en el surgimiento de ríos, quebradas, cochas y de espacios para los cultivos.

Tal vez por eso los kukama continúan manejando diversos recursos en el área de la llanura de inundación. El manejo y cultivo de las plantas es ordenado conforme avanzan las curvas de niveles en el terreno. Por ejemplo, notamos que, en el período de la seca, los kukama cultivan a las orillas del río Marañón el arroz, seguido de los frejoles. Conforme se va avanzando la topografía del terreno en dirección a la restinga, tendremos la presencia de los cultivos de plátano, maíz, maní y pimienta. Estos cultivos, la gran mayoría de veces, no se encuentran muy distantes de los núcleos residenciales, dado que se localizan detrás de las casas. Los kukama saben distinguir muy bien las plantas domesticadas, las semidomesticadas y las silvestres; estas últimas son aún utilizadas por médicos, chamanes y vegetalistas de las comunidades. El manejo de las plantas es tan funcional que, en la escuela de la comunidad Dos de Mayo, los estudiantes confeccionaron placas para identificar el nombre de las plantas nativas en la lengua kukama, con lo que se pretende valorizar el entorno a partir de vínculos de territorialidad.

Figura 4. Planta domesticada taya tini (paquitina blanca $^{6}$ ), utilizada para proteger la casa de los espíritus malos

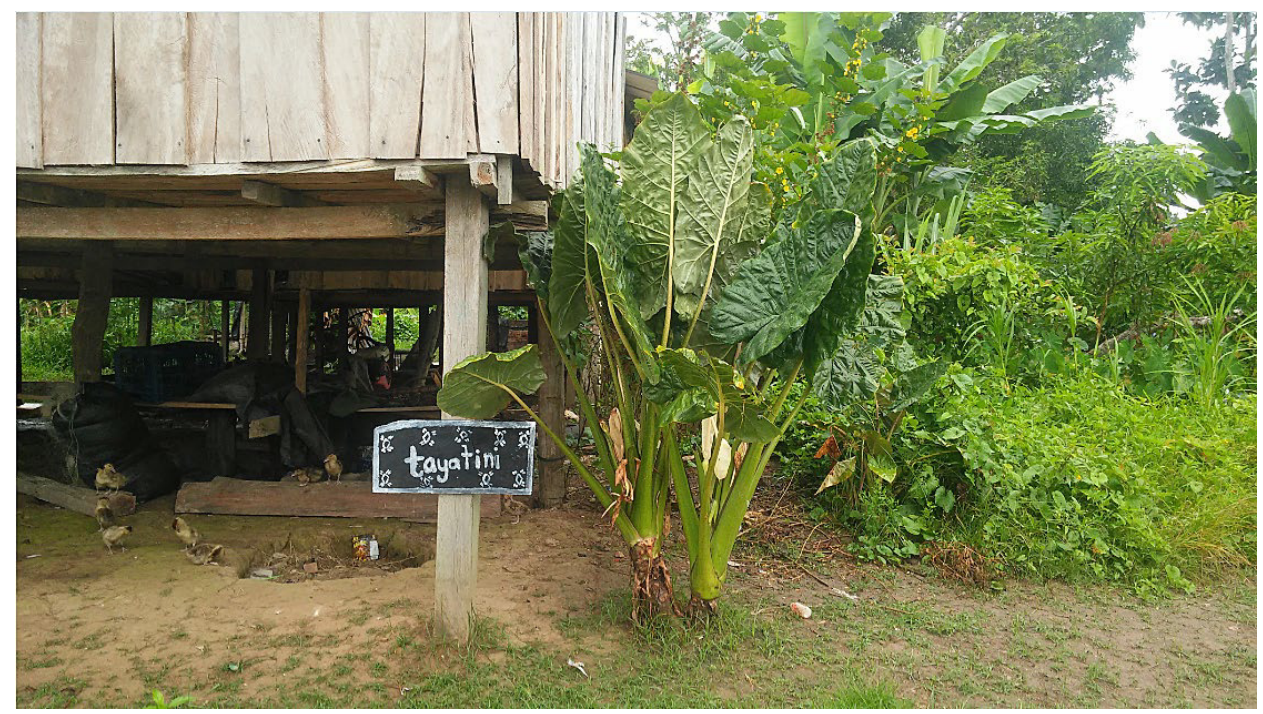

Fotografía: Marco Ramírez Colombier, octubre de 2017.

${ }^{6}$ Dieffenbachia obliqua. 
Por otra parte, la cocha Dos de Mayo es un lugar privilegiado para la pesca, actividad que sustenta la economía y la alimentación de las familias. Se calcula que cada persona en Dos de Mayo consume unos $430 \mathrm{~g}$ de pescado al día, distribuidos en dos comidas diarias (Rojas y Shuña, 2005). Según las historias contadas por las personas mayores, los fundadores de la comunidad, que llegaron como parte de expediciones caucheras en las primeras décadas del siglo XX, decidieron asentarse en esta zona debido a la abundancia de pescado, que les posibilitaba asegurar sus medios de vida.

Sin embargo, la presencia del pescado y, por consiguiente, el asentamiento de estos visitantes caucheros dependieron del hecho anterior de la formación de la cocha a través del movimiento de los ríos, que, como ya hemos visto, se debería al movimiento de la purawa. La existencia de la cocha San Pablo como lugar de pesca ha sido condicionada por la transformación territorial. Como cuenta en su siguiente testimonio Celso Manuyama, hijo de uno de los fundadores de la comunidad:

Esto se ha formado la cocha en el año 1910, aquí está ya registrado lo que han fundado, lo que han formado es en el año 1915 [...] [¿¿Cómo se forma la cocha?] Se ha trozado, el barranco le ha trozado por acá, y se quedó cocha. Por ahí es el Marañón ya. [¿̨Por qué sus padres fundaron el pueblo?] Por el pescado, acá era un mercado de peje, puro peje grande no chiquitito. Gamitana, zúngaro, paiche ${ }^{7}$, de esa manera vienen a vivir acá. Mi papá les ha gustado esa cocha porque había bastante peje, han venido acá y han tenido su familia. Han visto cómo ha formado la cocha. [¿Cómo le ha barranqueado?] Cuando era Marañón, esto era ungurahual, chambiral ${ }^{8}$, todito esto, tierra era allá, el barranco la restinga más alta le ha llevado el barranco, el mal paso era ahí este medio, las balsas pasaban por allá, para que pasen un bullonón era, tragadero era, muyuna $a^{9}$ lo que le dicen, entonces el balsero bogaba por el otro lado, pasaba por la playa. La muyuna una vez te jala ya no sales, ahí quedas. (Celso Manuyama, noviembre de 2017)

Considerando que esta breve historia presenta una serie de transformaciones a partir de relaciones sensoriales y rutas de posicionamiento en el espacio, podemos sugerir que las interacciones de los kukama con el agua, entre ellas la pesca, están cubiertas de sentidos de afectividad, historicidad y simbología.

Si bien los pescadores de Dos de Mayo pueden pescar en los distintos espacios de la cocha San Pablo, los lugares predilectos para pescar son las cochas Lorenzo y Mercado (esta última se llama así por la abundancia de pescado), que se encuentran a menos de quince minutos en bote de motor. Además de producir beneficios económicos y nutricionales fundamentales, la pesca es una actividad muy valorada por los kukama.

Colossoma macropomum, Zungaro zungaro y Arapaima gigas.

8 Zonas de crecimiento de Oenocarpus bataua y Astrocaryum chambira.

9 La muyuna es el término local para el remolino, peligroso fenómeno que aparece en las aguas del río. Los kukama lo relacionan con la boca abierta de la purawa. 
Los padres enseñan a sus hijos desde muy pequeños a controlar la canoa y lanzar las redes. Las salidas a pescar suelen ser un esfuerzo familiar, donde participan el padre, la madre y los niños. Ellos se ordenan en la canoa de la siguiente manera: el padre va en la proa, dirigiendo la embarcación con su remo; la madre va en la popa; y los niños, al medio. Cabe mencionar que los niños y las niñas kukama aprenden a manejar las canoas desde muy pequeños, lo que contribuye a su autonomía (necesitan la canoa para desplazarse por la comunidad inundada en invierno) y su crecimiento personal.

Algunos pescadores prefieren salir a pescar en las primeras horas de la mañana, mientras que otros prefieren la noche. En ambos casos, pero sobre todo de noche, la pesca se convierte en una actividad basada en el sentido del oído, pues cada especie de pescado hace un sonido distintivo cuando se mueve cerca de la superficie. Pescar requiere una sutileza estratégica basada en escuchar el agua:

Hay que escuchar al peje, no vas a largar tu trampa por largar. El pescado es estratégico, es mañoso también. Si una bulla que se hace... un poco de bulla y el peje se larga, silencio se queda, entonces tienes que escucharle para que puedas cazar al peje. Por eso es en la noche, ahorita no le ves, en la noche le escuchas bien, cómo boya. Ya sabes qué peje es, todo tipo de peje tiene su forma de boyar. (Teddy Manuyama, noviembre de 2017)

Figura 5. Secado al sol de pescado junto a las casas de la comunidad

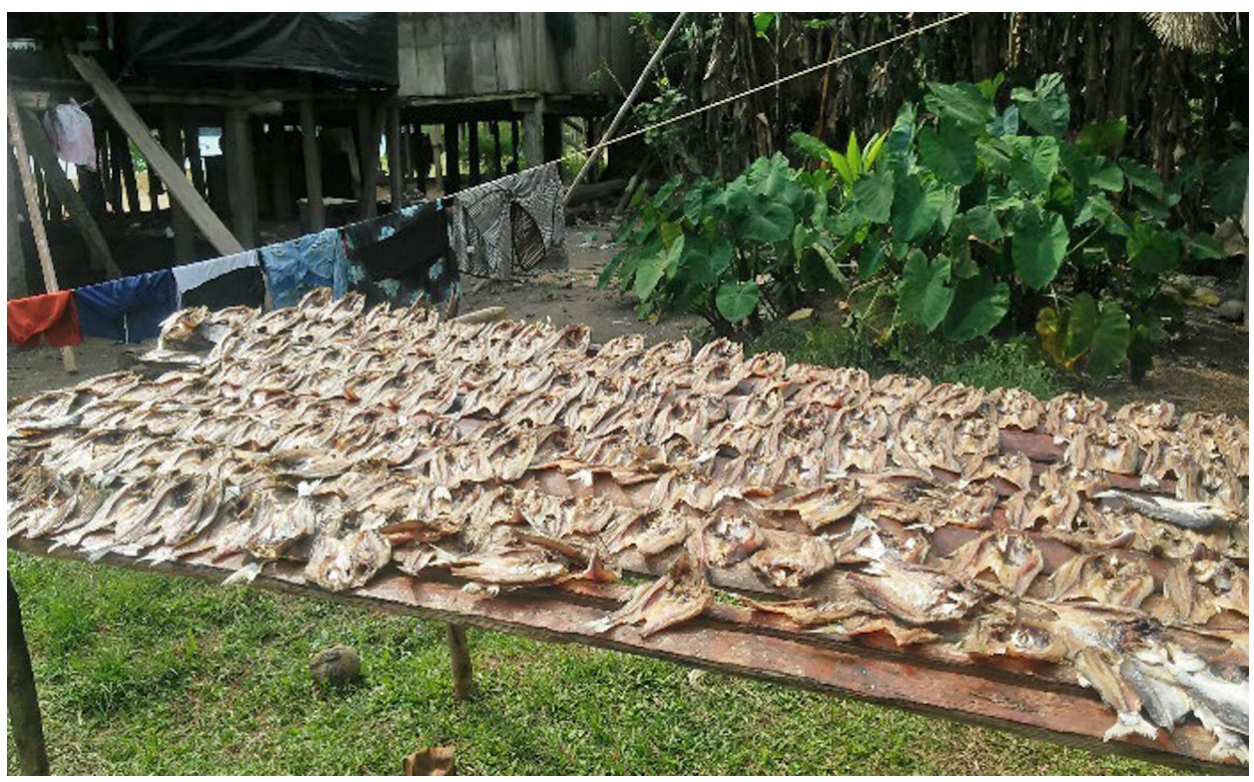

Fotografía: Marco Ramírez Colombier, julio de 2018. 
Figura 6. Nickson Barbarán, pescador de Dos de Mayo, mientras prepara los instrumentos de pesca con su hijo mayor

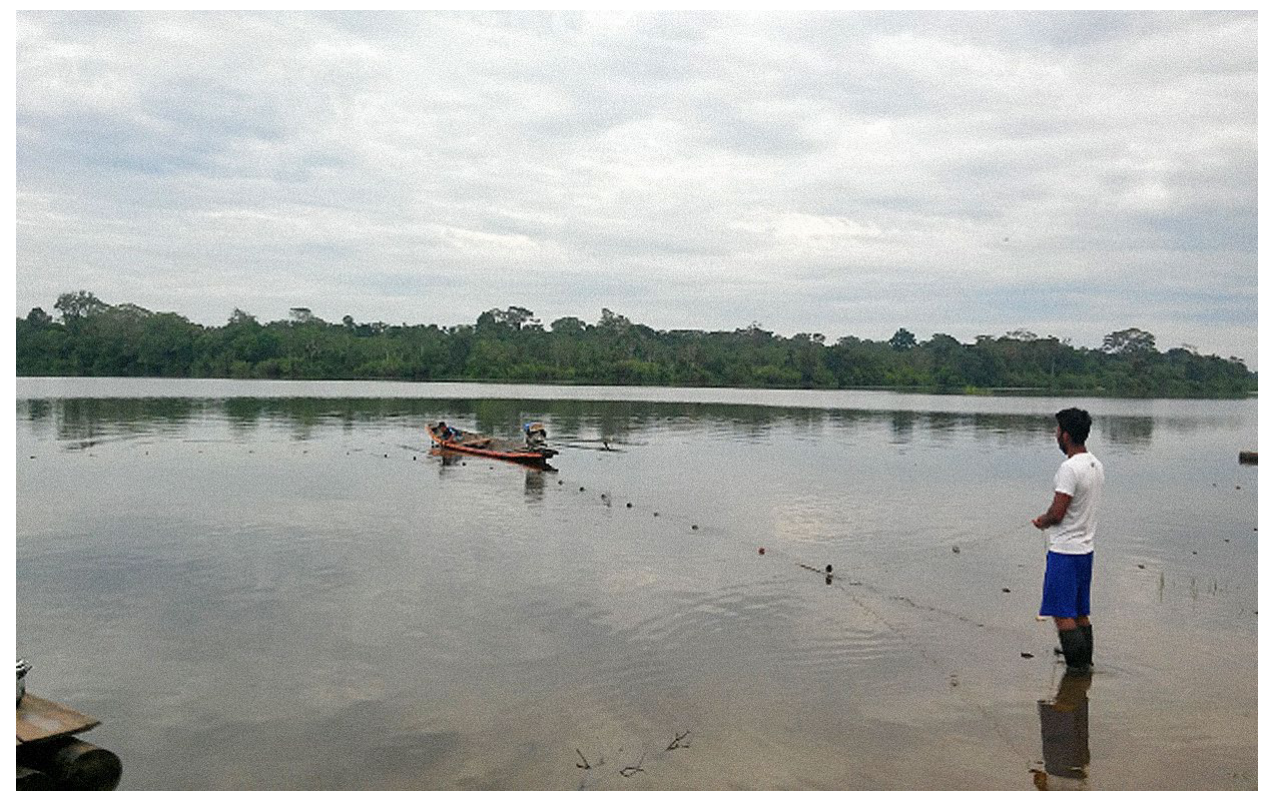

Fotografía: Marco Ramírez Colombier, julio de 2018.

Es preciso mencionar que las cochas y tahuampas (bosques inundados), espacios predilectos de pesca, son también lugares de aparición de seres subacuáticos, tanto pacíficos como depredadores, como el pelacara, una figura amenazante que ataca a las personas desprotegidas. Los pescadores que pasan la noche en el monte, cerca de las cochas, suelen tener sueńos significativos, donde pueden transportarse al mundo subacuático, comunicarse con los familiares, o interactuar con boas, sirenas y karwaras, habitantes de las ciudades bajo el agua. Estos seres son los dueños de los espacios acuáticos; los han domesticado y adecuado a sus necesidades, por lo que entrar a estos requiere una negociación por parte del pescador kukama. Vemos así que la domesticación y la territorialidad no son procesos exclusivos de los seres humanos.

\section{REgÍMENES DE CONSTRUCCIÓN DE ESPACIOS-TERRITORIOS}

Las descripciones de los espacios presentados nos llevan a pensar que, en la perspectiva kukama, su mundo está compuesto por una geografía afectiva totalmente diferente de los modelos antropocéntricos. En este contexto, interactúan categorías de gentes (Tello Imaina, 2016) responsables de tejer diversos modos de habitar entre los agentes humanos y no humanos (Fernandes Moreira, en preparación). Estas maneras de habitar, 
a su vez, contribuyen con la transformación de los espacios acuáticos y terrestres (Ramírez Colombier, 2018) debido a prácticas de domesticación y territorialidad. Sin embargo, el territorio kukama es rápidamente transformado, fuera de estas interacciones, por el impacto de los proyectos extractivos y de infraestructura, de la extracción de madera, del incremento de comercio y turismo, entre otros factores socioeconómicos. Incluso vemos que, en la misma comunidad, han llegado recientemente varias cabezas de ganado porcino y vacuno que amenazan con afectar el territorio comunal.

En las discusiones sobre el espacio y el territorio en la Amazonía, los mapas se han convertido en una herramienta poderosa, generada por el conocimiento del terreno y que sustenta regímenes de gobierno sobre la tierra que pueden determinar las vidas de las gentes de la cosmología kukama. Pensar el territorio desde la geografía afectiva y la información etnográfica del involucramiento de los kukamas y otros seres con el entorno nos lleva a considerar a los mapas generados por el Estado y otros actores de poder como herramientas que pretenden fijar el territorio y presentarlo como un entorno estático, a fin de poder controlarlo. Como menciona Ingold (2000), el ejercicio de cartografiar supone mirar el entorno "desde arriba», a partir de un imaginario punto perpendicular a la tierra, en un proceso muy distinto del ejercicio de búsqueda de caminos que realiza el cuerpo conocedor que se desplaza sobre un entorno con el que interactúa permanentemente.

Es así como en el entorno kukama del bajo Marañón coexisten distintos tipos de mapas: el mapa de la Reserva Nacional Pacaya Samiria, que determina los «bordes» de esta y de aquellos lugares donde el pueblo kukama no puede ver su autonomía de hecho reflejada en un título de propiedad legal sobre su territorio, dado que este espacio pertenece al Estado; los mapas del territorio comunal que algunas comunidades elaboraron con apoyo de la cooperación internacional, con el fin de acceder a títulos de propiedad; y el mapa en construcción que realiza la Hidrovía Amazónica, que opera bajo la premisa de una Amazonía desconectada y vacía, al mismo tiempo que invisibiliza los impactos negativos sobre el ecosistema que este proyecto puede generar.

En contraste con estos mapas, existen otras iniciativas que buscan generar otro tipo de discurso geográfico que ayude a exponer la complejidad y dinamismo del territorio kukama. Encontramos, por ejemplo, el mapa del río Marañón desarrollado por Radio Ucamara a partir de los testimonios de los moradores de las comunidades kukama, que registra puntos de interacción con los seres del río ${ }^{10}$, y las iniciativas de mapeo de puntos etnoarqueológicos y cosmológicos que desarrolla Daniel Fernandes Moreira, también con apoyo de los moradores. Como observamos, los mapas son dispositivos

\footnotetext{
${ }^{10}$ Para más información, véase Mcarbajal. (26 de setiembre, 2016). Mapeo cultural, espiritual, territorial del Pueblo Kukama (Bajo río Marañón). WCS Perú. Recuperado de https://peru.wcs.org/es-es/WCSPer\%C3\%BA/Noticias/articleType/ArticleView/articleId/9297/Mapeo-cultural-espiritual-territorial-delPueblo-Kukama-Bajo-rio-Maranon.aspx.
} 
para generar discursos sobre la geografía local, con el fin político de la defensa del territorio, y no necesariamente herramientas para guiarse dentro de esta.

El discurso geográfico de los «mapas afectivos» apunta a superar las limitaciones que ofrece el concepto de espacio y a establecer el concepto de territorio como un todo integral de interacciones complejas entre seres y mundos, que nos invita a superar el discurso naturalista sobre la Amazonía (Descola, 2014), y que busca sintonizar con la necesidad de reconocimiento de los derechos de los pueblos indígenas (Surrallés, 2009) y de protección de su legitimidad territorial (Fernandes Moreira, 2012; Virtanen, Stoll y Tola, 2017). Mientras que el Estado y las empresas de infraestructura pretenden estabilizar el espacio para que no pueda tornarse territorio, los pueblos indígenas plantean maximizar sus transformaciones a través de la acción humana y construyen, así, entornos domesticados donde puedan ejercer sus autonomías. En el plano teórico, esto nos advierte de la necesidad de involucrar estas prácticas indígenas, basadas en los sentidos, el cuerpo y las emociones, en la teoría geográfica y de las demás ciencias sociales para fortalecer el tránsito desde las descripciones y explicaciones propias de la geografía ambiental hacia el crecimiento de una geografía afectiva.

\section{REFERENCIAS BIBLIOGRÁFICAS}

Acodecospat, Aidecos, Akubana, Huaynakana Kamatawara Kana y Orpio (2015). Proceso de consulta de proyecto Hidrovía. Acta de la evaluación interna pueblo kukama. Reunión en Nauta, del 10 al 12 de julio de 2015. Recuperado de http:// observatoriopetrolero.org/wp-content/uploads/2015/07/Acta-Evaluaci\%C3\%B3nInternaKukama_Hidrovia.pdf

Ames, P. (2010). Desigualdad y territorio en el Perú: una geografía jerarquizada. Revista Argumentos, (1). Recuperado de http://revistaargumentos.iep.org.pe/articulos/ desigualdad-y-territorio-en-el-peru/

Balée, W. (2013). Cultural forests of the Amazon: a historical ecology of people and their landscapes. Tuscaloosa, EEUU: University of Alabama Press.

Balée, W. y Erickson, C. (2006). Time, complexity, and historical ecology. En W. Balée y C. Erickson (eds.), Time and complexity in historical ecology: studies in the Neotropical Lowlands (pp. 1-20). Nueva York, EE.UU.: Columbia University Press. https://doi. org/10.7312/bale13562

Berjón, M. y Cadenas, M. A. (2014). Inestabilidad ontológica: el caso de los kukama de la Amazonía peruana. Publicado por la Organización de Agustinos de Latinoamérica (OALA). Recuperado de http://www.oalagustinos.org/pdf/2014_15Manuel.pdf

Bristow, T. (2015). The Anthropocene lyric. An affective geography of poetry, person and place. Londres, Reino Unido: Palgrave Macmillan UK. https://doi.org/ $10.1057 / 9781137364753$ 
Campanera Reig, M. (2017). De lagos propios a patrimonio de la Nación. Disputas por el espacio acuático en la Reserva Nacional Pacaya Samiria. Revista de Antropología Social, 26(2), 281-306. https://doi.org/10.5209/RASO.57607

Carvajal, G. de (2010 [1540]). Descubrimiento del río de las Amazonas. Biblioteca Virtual Universal. Recuperado de http://www.biblioteca.org.ar/libros/153797.pdf

Castro Ríos, M. (2015). Una aproximación al proceso de afirmación étnica del pueblo cocama: el caso del caserio de Santo Tomás (Tesis de licenciatura). Lima, Perú: Pontificia Universidad Católica del Perú.

Cayón, L. (2014). Planos de vida e manejo do mundo. Revista de Estudos em Relaçóes Interétnicas, 18(1), 1-21. https://doi.org/10.26512/interethnica.v18i1.15371

Chibnik, M. (1994). Risky rivers: the economics and politics of floodplain farming in Amazonia. Tucson, EEUU: University of Arizona Press.

Clement, Ch. R., Denevan, W. M., Heckenberger, J., Braga Junqueira, A., Neves, E. G., Teixeira, W. G. y Woods, W. I. (2015). The domestication of Amazonia before European conquest. Proceedings of the Royal Society B: Biological Sciences, 282(1812). https://doi.org/10.1098/rspb.2015.0813

De la Cadena, M. (2010). Indigenous cosmopolitics in the andes: conceptual reflections beyond «politics». Cultural Anthropology, 25(2), 334-370. https://doi.org/10.1111/ j.1548-1360.2010.01061.x

Descola, P. (1986). La selva culta: simbolismo y praxis en la ecología de los achuar. Quito, Ecuador: Instituto Francés de Estudios Andinos, Ediciones Abya-Yala.

Descola, P. (1996). Constructing natures: symbolic ecology and social practice. En P. Descola y G. Pálsson (eds.), Nature and society: anthropological perspectives (pp. 82-102). Londres, Reino Unido: Routledge.

Descola, P. (2014). ¿Existen paisajes amazónicos? En S. Rostain (ed.), Amazonía. Memorias de las conferencias magistrales del III Encuentro Internacional de Arqueología Amazónica (pp. 19-30). Quito, Ecuador: Ministerio Coordinador de Conocimiento y Talento Humano e IKIAM, Secretaría Nacional de Educación Superior, Ciencia, Tecnología e Innovación, Tercer Encuentro Internacional de Arqueología Amazónica.

Dillehay, T. (2007). Concepts of time, space, creation narratives, and knowledge. En T. Dillehay, Monuments, empires, and resistance: the Araucanian polity and ritual narratives (pp. 171-177). Cambridge, EEUU: Cambridge University Press. https://doi. org/10.1017/CBO9780511499715

Echeverri, J. A. (2004). Territorio como cuerpo y territorio como naturaleza: ¿diálogo intercultural? En A. Surrallés y P. García Hierro (eds.), Tierra adentro. Territorio indigena y percepción del entorno (pp. 259-276). Lima, Perú: IGWIA.

Espinosa de Rivero, O. (2009). ¿Salvajes opuestos al progreso?: aproximaciones históricas y antropológicas a las movilizaciones indígenas en la Amazonía peruana. Anthropologica, 27(27), 123-168. 
Espinosa de Rivero, O. (2010). Cambios y continuidades en la percepción y demandas indígenas sobre el territorio en la Amazonía peruana. Anthropologica, 28(28), 239-262.

Fernandes Moreira, D. (2012). Patrimônio cultural da Amazônia Ocidental Brasileira: Na defesa da Educação Patrimonial diante da fragmentação e resistência da comunidade de Jacy-Paraná (RO) (Tesis de maestría). Porto Velho, Brasil: Universidade Federal de Rondônia.

Fernandes Moreira, D. (En preparación). La "Gente» en los Mundos: modos de habitar Kukama en la Amazonía peruana (Tesis doctoral). Lima, Perú: Pontificia Universidad Católica del Perú.

Fernandes Moreira, D. y Ramírez Colombier, M. (2019). Mi casa pequeña, mi corazón grande. Política territorial y cosmológica del pueblo kukama. Mundo Amazónico, 10(1). https://doi.org/10.15446/ma.v10n1.73980

García Hierro, P. (2004). Territorios indígenas: tocando a las puertas del Derecho. En A. Surrallés y P. García Hierro (eds.), Tierra adentro. Territorio indígena y percepción del entorno (pp. 277-306). Lima, Perú: IGWIA.

Garra, S. y Riol Gala, R. (2014). Por el curso de las quebradas hacia el 'territorio integral indígena': autonomía, frontera y alianza entre los awajún y wampis. Anthropologica, 32(32), 41-70.

Heckenberger, M. y Neves, E. G. (2009). Amazonian archaeology. Annual Review of Anthropology, 38, 251-266. https://doi.org/10.1146/annurev-anthro-091908-164310

Huaymacari Tamani, J. M. (2016). Cómo aparecieron los ríos, cochas y quebradas. En L. Tello Imaina y S. Boyd (eds.), J. M. Huaymacari Tamani y M. Nieves Nashnato (trads.), Karuara: la gente del río (pp. 97-104). Cusco, Iquitos, Parinari (Perú): Asociación Quisca, Radio Ucamara, Wainakana Kamatawarakana.

Ingold, T. (2000). The perception of the environment. Nueva York, EE.UU./Londres, Reino Unido: Routledge.

Karadimas, D., Lécrivain, V. y Rostain, S. (eds.). (2018). De l'ethnologie à la préhistoire. París, Francia: L'Herne. Cahiers d'Anthropologie Sociale.

Lathrap, D. W. (1970). The upper Amazon. Berkeley, EE.UU./Londres, Reino Unido: Thomes and Hudson.

Meggers, B. (1976). Amazonía. Hombre y cultura en un paraíso ilusorio. México DF, México: Siglo XXI.

Miller, E. Th. (1987). Pesquisas arqueológicas Paleoindígenas no Brasil Ocidental. Estudios Atacameños, (8), 37-61. https://doi.org/10.22199/S07181043.1987.0008.00005

Morales Chocano, D. (2002). Contactos entre cocamas y shipibos: un acercamiento arqueológico en la Amazonía peruana. Investigaciones Sociales, 6(10), 47-70. https:// doi.org/10.15381/is.v6i10.8092

Neves, E. G. (2011). El nacimiento del presente etnográfico: la emergencia del patrón de distribución de sociedades indígenas y familias lingüísticas en las tierras bajas 
sudamericanas, durante el primer milenio d. C. En J.-P. Chaumeil, O. Espinosa de Rivero y M. Cornejo Chaparro (eds.), Por donde hay soplo. Estudios amazónicos en los paises andinos (pp. 39-65). Lima, Perú: IFEA, PUCP, CAAAP, EREA-LESC.

Okamoto T. y Leifsen E. (2012). Oil spills, contamination, and unruly engagements with indigenous peoples in the Peruvian Amazon. En H. Haarstad (ed.), New political spaces in Latin American natural resource governance. Studies of the Americas. Nueva York, EE.UU.: Palgrave Macmillan. https://doi.org/10.1057/9781137073723_9

Padoch, C., Brondizio, E., Costa, S., Pinedo-Vasquez, M., Sears, R. R. y Siqueria, A. (2008). Urban forest and rural cities: multi-sited households, consumption patterns, and forest resources in Amazonia. Ecology and Society, 13(2). https://doi.org/10.5751/ ES-02526-130202

Pile, S. (2009). Emotions and affect in recent human geography. Transactions, 35(1), 5-20. https://doi.org/10.1111/j.1475-5661.2009.00368.x

Politis, G. (2007). Nukak. Ethnoarchaeology of an Amazonian people. Walnut Creek, CA: Left Coast Press.

Ramírez Colombier, M. (2018). Cuerpos y territorialidad del pueblo kukama en la política contemporánea sobre la Amazonía (Tesis de maestría). Lima, Perú: Pontificia Universidad Católica del Perú.

Rivas, R. (2004). El gran pescador. Técnicas de pesca entre los cocama-cocamillas de la Amazonia peruana. Lima, Perú: Pontificia Universidad Católica del Perú.

Rivas, R. (2011). Le serpent, mère de l'eau. Chamanisme aquatique chez les Cocama-Cocamilla d'Amazonie péruvienne (Tesis doctoral). París, Francia: École des Hautes Études en Sciences Sociales.

Rojas, J. y Shuña, R. (2005). Estado situacional de la pesquería comunal y sus parámetros socio-económicos en la cocha San Pablo de Tipishca, Reserva Nacional Pacaya Samiria (Tesis de licenciatura). Iquitos, Perú: Universidad Nacional de la Amazonía Peruana.

Roosevelt, A. (1993). The rise and fall of the Amazon chiefdoms. L'Homme, 33(126/128), 255-283. https://doi.org/10.3406/hom.1993.369640

Rostain, S. y Jaimes Betancourt, C. (eds.). (2017). Las siete maravillas de la Amazonía precolombina. La Paz, Bolivia: Plural Editores.

Santos Angarita, A. (2013). Percepción tikuna de Naane y Naüne: territorio y cuerpo (Tesis de maestría). Leticia, Colombia: Universidad Nacional de Colombia Sede Amazonía.

Santos-Granero, F. (1998). Writing History into the landscape: space, myth, and ritual in contemporary Amazonia. American Ethnologist, 25(2), 128-148. https://doi. org/10.1525/ae.1998.25.2.128

Silva, F. A. (2014). El pasado en el presente de los Asurini do Xingu: narrativas arqueológicas y narrativas indígenas. En M. C. Rivolta, M. Montenegro, L. Menezes Ferreira (eds.), Multivocalidad y activaciones patrimoniales en arqueologia: perspectivas desde 
Sudamérica (pp. 131-154). Buenos Aires, Argentina: Fundación de Historia Natural Félix de Azara.

Stocks, A. (1981). Los nativos invisibles. Notas sobre la historia y realidad actual de los cocamilla del rio Huallaga, Perú. Lima, Perú: CAAAP.

Surrallés, A. (2004). Horizontes de intimidad. Persona, percepción y espacio en los Candoshi. En A. Surrallés y P. García Hierro (eds.), Tierra adentro. Territorio indígena y percepción del entorno (pp. 137-162). Lima, Perú: IGWIA.

Surrallés, A. (2014). Entre derecho y realidad: antropología y territorios indígenas amazónicos en un futuro próximo. Bulletin de l'Institut Français d'études Andines, 38(1), 29-45. https://doi.org/10.4000/bifea.2789

Tello Imaina, L. (2014). Ser gente en la Amazonía, fronteras de lo humano: aporte del pueblo kukama. En R. Badini (ed.), Amazzonia indigena e pratiche di autorappresentazione (pp. 39-48). Milán, Italia: FrancoAngeli.

Tello Imaina, L. (2016). «Prólogo». En L. Tello Imaina y S. Boyd (eds.), J. M. Huaymacari Tamani y M. Nieves Nashnato (trads.), Karuara: la gente del río (pp. 8-10). Cusco, Iquitos, Parinari (Perú): Asociación Quisca, Radio Ucamara, Wainakana Kamatawarakana.

Uzendoski, M. A. (2012). Beyond orality. Textuality, territory, and ontology among Amazonian peoples. HAU: Journal of Ethnographic Theory, 2(1). https://doi.org/10.14318/ hau2.1.005

Vadjunec, J. M., Schmink, M. y Greiner, A. L. (2011). New Amazonian geographies: emerging identities and landscapes. Journal of Cultural Geography, 28(1), 1-20. https:// doi.org/10.1080/08873631.2011.548477

Virtanen, P. K., Stoll, E. y Tola, F. (2017). Les vestiges comme source de légitimation territoriale. Nuevo Mundo Mundos Nuevos. https://doi.org/10.4000/nuevomundo.70144

Viveiros de Castro, E. (1996). Images of nature and society in Amazonian ethnology. Annual Review of Anthropology, 25(1), 179-200. https://doi.org/10.1146/annurev. anthro.25.1.179

Viveiros de Castro, E. (1998). Cosmological deixis and Amerindian perspectivism. The Journal of the Royal Anthropological Institute, 4(3), 469-488. https://doi. org/10.2307/3034157

Zanotti, L. (2018). Political ecology of movement: trekking and territoriality among the Kayapó. Journal of Political Ecology, 21(1), 108-126. https://doi.org/10.2458/ v21i1.21127 